\title{
Glial-Specific Deletion of Med12 Results in Rapid Hearing Loss via Degradation of the Stria Vascularis
}

\author{
${ }^{\circ}$ Teng-Wei Huang, ${ }^{1}$ Amrita A. Iyer, ${ }^{3,6}$ Jeanne M. Manalo, ${ }^{4}$ Junsung Woo, ${ }^{1}{ }^{\circledR}$ Navish A. Bosquez Huerta, ${ }^{1,5}$ \\ Melissa M. McGovern, ${ }^{2}$ Heinrich Schrewe, ${ }^{7}{ }^{\circledR}$ Fredrick A. Pereira, ${ }^{8,9}{ }^{\circledR}$ Andrew K. Groves, ${ }^{2,3,5,6}$ \\ Kevin K. Ohlemiller, ${ }^{10}$ and Benjamin Deneen ${ }^{1,2,5,11}$ \\ ${ }^{1}$ Center for Cell and Gene Therapy, Baylor College of Medicine, Houston, Texas 77030, ${ }^{2}$ Department of Neuroscience, Baylor College of Medicine, \\ Houston, Texas 77030, ${ }^{3}$ Department of Molecular and Human Genetics, Baylor College of Medicine, Houston, Texas 77030, ${ }^{4}$ Department of \\ Biochemistry and Molecular Biology, The University of Texas Health Science Center at Houston, Houston, Texas $77030,{ }^{5}$ Program in \\ Developmental Biology, Baylor College of Medicine, Houston, TX 77030, ${ }^{6}$ Program in Genetics \& Genomics, Baylor College of Medicine, Houston, \\ Texas 77030, ${ }^{7}$ Department of Developmental Genetics, Max Planck Institute for Molecular Genetics, 14195 Berlin, Germany, ${ }^{8}$ Huffington Center on \\ Aging, Baylor College of Medicine, Houston, Texas 77030, ${ }^{9}$ Department of Otolaryngology, Baylor College of Medicine, Houston, Texas 77030, \\ ${ }^{10}$ Department of Otolaryngolgy, Central Institute for the Deaf, Fay and Carl Simons Center for Biology of Hearing and Deafness, Washington \\ University in St. Louis, St. Louis, Missouri 63110, and ${ }^{11}$ Department of Neurosurgery, Baylor College of Medicine, Houston, Texas 77030
}

Mediator protein complex subunit 12 (Med12) is a core component of the basal transcriptional apparatus and plays a critical role in the development of many tissues. Mutations in Med12 are associated with X-linked intellectual disability syndromes and hearing loss; however, its role in nervous system function remains undefined. Here, we show that temporal conditional deletion of Med12 in astrocytes in the adult CNS results in region-specific alterations in astrocyte morphology. Surprisingly, behavioral studies revealed rapid hearing loss after adult deletion of Med12 that was confirmed by a complete abrogation of auditory brainstem responses. Cellular analysis of the cochlea revealed degeneration of the stria vascularis, in conjunction with disorganization of basal cells adjacent to the spiral ligament and downregulation of key cell adhesion proteins. Physiologic analysis revealed early changes in endocochlear potential, consistent with strial-specific defects. Together, our studies reveal that Med12 regulates auditory function in the adult by preserving the structural integrity of the stria vascularis.

Key words: astrocyte; hearing loss; mediator complex; stria vascularis

Significance Statement

Mutations in Mediator protein complex subunit 12 (Med12) are associated with X-linked intellectual disability syndromes and hearing loss. Using temporal-conditional genetic approaches in CNS glia, we found that loss of Med12 results in severe hearing loss in adult animals through rapid degeneration of the stria vascularis. Our study describes the first animal model that recapitulates hearing loss identified in Med12-related disorders and provides a new system in which to examine the underlying cellular and molecular mechanisms of Med12 function in the adult nervous system.

\section{Introduction}

Glial cell support of neuronal function is a hallmark feature of nervous systems across evolution, with both glial abundance and

Received Jan. 12, 2021; revised June 11, 2021; accepted July 5, 2021.

Author contributions: T.-W.H., J.W., K.K.O., and B.D. designed research; T.-W.H., A.A.I., J.M.M., J.W., N.A.B.H., and K.K.O. performed research; A.A.I., J.M.M., M.M.M., H.S., and A.K.G. contributed unpublished reagents/analytic tools; T.-W.H., A.A.I., N.A.B.H., F.A.P., and A.K.G. analyzed data; T.-W.H. and B.D. wrote the paper.

This work was supported by grants from the National Multiple Sclerosis Society (FG-1607-25417, to

T.-W.H.) and the National Institutes of Health (NS-071153 and NS-096096, to B.D.; and DC-014832 to A.K.G.).

A.A.I. was supported in part by The Cullen Foundation.

The authors declare no competing financial interests.

Correspondence should be addressed to Benjamin Deneen at deneen@bcm.edu.

https://doi.org/10.1523/JNEUROSCI.0070-21.2021

Copyright $\odot 2021$ the authors specialization correlated with increasing neuronal and organism complexity (Freeman and Rowitch, 2013). Accordingly, mammalian systems contain a host of specialized glial cells that subserve neuronal function, with astrocytes serving as an archetype for glial support (Allen and Lyons, 2018; Nagai et al., 2021). Among the key functions performed by astrocytes include buffering neurotransmitters, maintenance of ion gradients, and providing metabolic support, all of which directly influence the function of neurons and associated circuits (Khakh and Deneen, 2019).

In the mammalian auditory system hair cells use mechanosensation to convert energy from soundwaves into neurophysiological signals that are transmitted to the brainstem (Brownell et al., 1985; Chan and Hudspeth, 2005; Muller, 2008). Hair cell 
function relies on the endocochlear potential (EP), which is an ion gradient established in the endolymph and maintained by the stria vascularis (Gulley and Reese, 1976; Patuzzi, 2011). The resting potential of the endolymph is regulated by the transport of $\mathrm{K}^{+}$ions across the stria vascularis into the cochlear duct (Gründer et al., 2001; Boettger et al., 2002; Mittal et al., 2017). This function is mediated by potassium channels, including Kir4.1, which is the predominant channel used by astrocytes to buffer neuronal $\mathrm{K}^{+}$(Rozengurt et al., 2003; Chen and Zhao, 2014). Critically, disruption of these electrochemical gradients in the endolymph can lead to altered hair cell function and survival, culminating in a loss of hearing (Nin et al., 2016; Mittal et al., 2017). While much progress has been made in understanding how the stria vascularis regulates endocochlear potential, the transcriptional mechanisms that control stria vascularis function remain poorly defined.

Glial development offers a venue in which to identify transcription factors that regulate mature glial cell function. For example, Sox10 and NFIA (nuclear factor IA) are key regulators of oligodendrocyte and astrocyte development, respectively, and the physiological functions of these cells in the adult CNS (Stolt et al., 2002; Huang et al., 2020). These parallels also apply to auditory support cells, where Sox2, Sox9, and Sox10 are expressed in developing and mature CNS glia and support cells in the cochlea (Watanabe et al., 2000; Oesterle et al., 2008; Loponen et al., 2011). Drawing on this parallel, we identified Mediator Complex Subunit 12 (Med12) as a developmental transcription factor that is enriched in subsets of astrocytes in the adult brain (John Lin et al., 2017). Studies in development have shown that Med12 cooperates with Sox10 to regulate oligodendrocyte differentiation (Vogl et al., 2013); however, its role in mature astrocytes is undefined. Furthermore, mutations in Med12 are linked to intellectual disability syndromes and are coupled with hearing loss (Schwartz et al., 2007; Lesca et al., 2013; Vulto-van Silfhout et al., 2013; Donnio et al., 2017; Rubinato et al., 2020). These observations implicate Med12 as a critical transcription factor contributing to a host of nervous systems functions related to neuronal circuits, myelination, and auditory function. Despite its widespread expression in neurons and glia, the precise role of Med12 in the adult CNS remains unknown.

To dissect the contributions of Med12 toward brain function, we generated temporal-conditional mouse knock-out (KO) models that enabled us to delete Med12 in astrocytes. Analysis across brain regions revealed reduced astrocyte complexity in the hippocampus, while behavioral studies revealed that mice lacking astrocytic-Med12 are deaf and have no auditory brainstem response (ABR). Cellular analysis of the cochlea revealed that Med12 is required to maintain the integrity of the stria vascularis, which eventually results in eventual loss of hair cells. Together, these studies identify Med12 in the stria vascularis as a key regulator of auditory function.

\section{Materials and Methods \\ Animals}

All experimental animals were treated in compliance with the US Department of Health and Human Services, the National Institutes of Health guidelines, and Baylor College of Medicine Institutional Animal Care and Use Committee (IACUC) guidelines. All mice were housed with food and water available ad libitum in a $12 \mathrm{~h}$ light/dark environment. Both female and male mice were used for all experiments, and littermates of the same sex were randomly allocated to experimental groups. For histologic analyses of brains and behavior assays, 4-monthold adult mice were used unless otherwise described. For ABR test and histologic analyses of inner ears, examinations or tissue collections were performed at 1,2,3, or 5 weeks after the tamoxifen (TM) treatment, as described. All mice used in this study were maintained on a C57BL/6J background. Med12 conditional knock-out mice were generated by crossing Med12 $2^{\mathrm{fl} / \mathrm{fl}}$ or Med $12^{\mathrm{fl} / \mathrm{y}}$ conditional mutant mice (Rocha et al., 2010a,b) with Aldh1l1-CreER (The Jackson Laboratory; RRID:IMSR JAX:029655), resulting in Med12 $2^{\mathrm{fl} / \mathrm{fl}}$; Aldh1l1-CreER or Med12 $2^{\mathrm{f} / \mathrm{y}}$; Aldh1l1-CreER (A12-KO) and Med12 $2^{\mathrm{f} / \mathrm{fl}}$ or Med12 $2^{\mathrm{fl} / \mathrm{y}}$ (A12-Con) littermate controls. For histologic analysis, the Aldh1l1-GFP mouse was crossed with the A12-KO mouse, resulting in Med12 $2^{\mathrm{fl} / \mathrm{fl}}$ (or Med12 $2^{\mathrm{fl} / \mathrm{y}}$ ); Aldh1l1-CreER; Aldh1l1-GFP (AG12-KO) and Med12 $2^{\mathrm{fl} / \mathrm{fl}}$ (or Med12 $2^{\mathrm{fl} / \mathrm{y}}$ ); Aldh1l1-GFP (AG12-Con) mice. To mark the Cre-targeted cells, RosaCAG-LSL-tdTomato-WPRE mice were crossed with A12-KO mice, resulting in Med1 $2^{\mathrm{fl} / \mathrm{fl}}\left(\right.$ or Med12 $\left.2^{\mathrm{f} / \mathrm{y}}\right)$; Aldh1l1-CreER; Rosa-CAG-LSLtdTomato-WPRE (AT12-KO) and Med12 $2^{+/+}$(or Med12 ${ }^{+/ y}$ ); and Aldh1l1-CreER; Rosa-CAG-LSL-tdTomato-WPRE (AT12-Con) mice. To induce deletion of Med12 in the adult, 4-week-old mice were gavage fed with $150 \mathrm{mg} / \mathrm{kg}$ body weight tamoxifen (catalog \#T5648, SigmaAldrich) dissolved in corn oil twice per day for $5 \mathrm{~d}$. The above experiments were approved by Baylor College of Medicine IACUC.

\section{Immunofluorescence on frozen tissue sections}

Mice were anesthetized under isoflurane inhalation and perfused transcardially with $1 \times$ PBS, pH 7.4, followed by $4 \%$ paraformaldehyde (PFA). Brains were removed, postfixed in $4 \%$ PFA for $8 \mathrm{~h}$, and placed in $20 \%$ sucrose for $16 \mathrm{~h}$ before being embedded in optimal cutting temperature (OCT) compound. Inner ears were dissected from temporal bones and fixed in 4\% PFA overnight, decalcified in 0.2 m EDTA for $24 \mathrm{~h}$. Decalcified inner ears were incubated in $7.5 \%$ gelatin solution $(1 \times \mathrm{PB} /$ $10 \%$ sucrose) at $37^{\circ} \mathrm{C}$ for $1 \mathrm{~h}$ before being embedded in OCT compound. Brain sections of $30 \mu \mathrm{m}$ and cochlea sections of $12 \mu \mathrm{m}$ were made on a cryostat. For immunostaining, tissue sections were washed with $1 \times$ PBS for 5 min three times, blocked with $10 \%$ goat serum in PBS with $0.3 \%$ Tween 20, and then incubated with primary antibodies in blocking solution overnight. On the next day, sections were incubated with secondary antibodies in PBS with $0.1 \%$ Tween 20 for $1 \mathrm{~h}$ at room temperature, followed by incubation with DAPI for $5 \mathrm{~min}$, and mounted with VECTASHIELD Antifade Mounting Media (catalog \#H-1000, Vector Laboratories). The following primary antibodies were used: chicken anti-GFP (1:1000; catalog \#ab13970, abcam); rabbit anti-Med12 (1:1000; catalog \#NB100-2357, Novus Biologicals); chicken anti-GFAP (1:1000; catalog \#ab4674, abcam); mouse anti-GFAP (1:1000; catalog \#MAB360, EMD Millipore); mouse anti-NeuN (1:500; catalog \#MAB377, Millipore); rabbit anti-myosin-VIIa (1:1000; catalog \#25-6790, Proteus BioSciences); mouse anti-myosin-VIIa (1:500; catalog \#MYO7A, Development Studies Hybridoma Bank); rabbit anti-Sox2 (1:1000; catalog \#AB5603, Millipore); rat anti-Sox2 (1:1000; catalog \#14-9811-82, Thermo Fisher Scientific); rabbit anti-GLUT1 (1:500; catalog \#ab115730, abcam); mouse anti-ZO-1 (1:500; catalog \#33-9100, Thermo Fisher Scientific); mouse anti-E-cadherin (Ecad; 1:200; catalog \#610181, BD Biosciences); and rabbit anti-connexin 31 (Cx31; 1:250; catalog \#12880-1-AP, Proteintech Group). The following secondary antibodies were used (1:500): Alexa Fluor 488 goat anti-chicken (catalog \#A11039, Thermo Fisher Scientific); Alexa Fluor 488 goat anti-mouse (catalog \#A32723, Thermo Fisher Scientific); Alexa Fluor 488 goat anti-rabbit (catalog \#A32731, Thermo Fisher Scientific); Alexa Fluor 568 goat anti-mouse (catalog \#A-11004, Thermo Fisher Scientific); Alexa Fluor 568 goat anti-rabbit (catalog \#A11036, Thermo Fisher Scientific); Alexa Fluor 647 goat anti-mouse (catalog \#A32728, Thermo Fisher Scientific); and Alexa Fluor 647 goat anti-rabbit (catalog \#A32733, Thermo Fisher Scientific).

\section{Confocal imaging and image analysis}

To measure astrocyte morphology, fluorescent images were acquired using a Zeiss LSM 880 laser scanning confocal microscope with $63 \times$ oilimmersion objective with frame size at $1024 \times 1024$ and bit depth at 12 . Serial images at $z$-axis were taken at an optical step of $1 \mathrm{~mm}$, with overall $z$-axis range encompassing the whole section. Images were imported to Imaris Bitplane software, and only astrocytes with their soma between the $z$-axis range were chosen for further analysis (Lanjakornsiripan et al., 2018). We performed 3D surface rendering using the Imaris Surface 

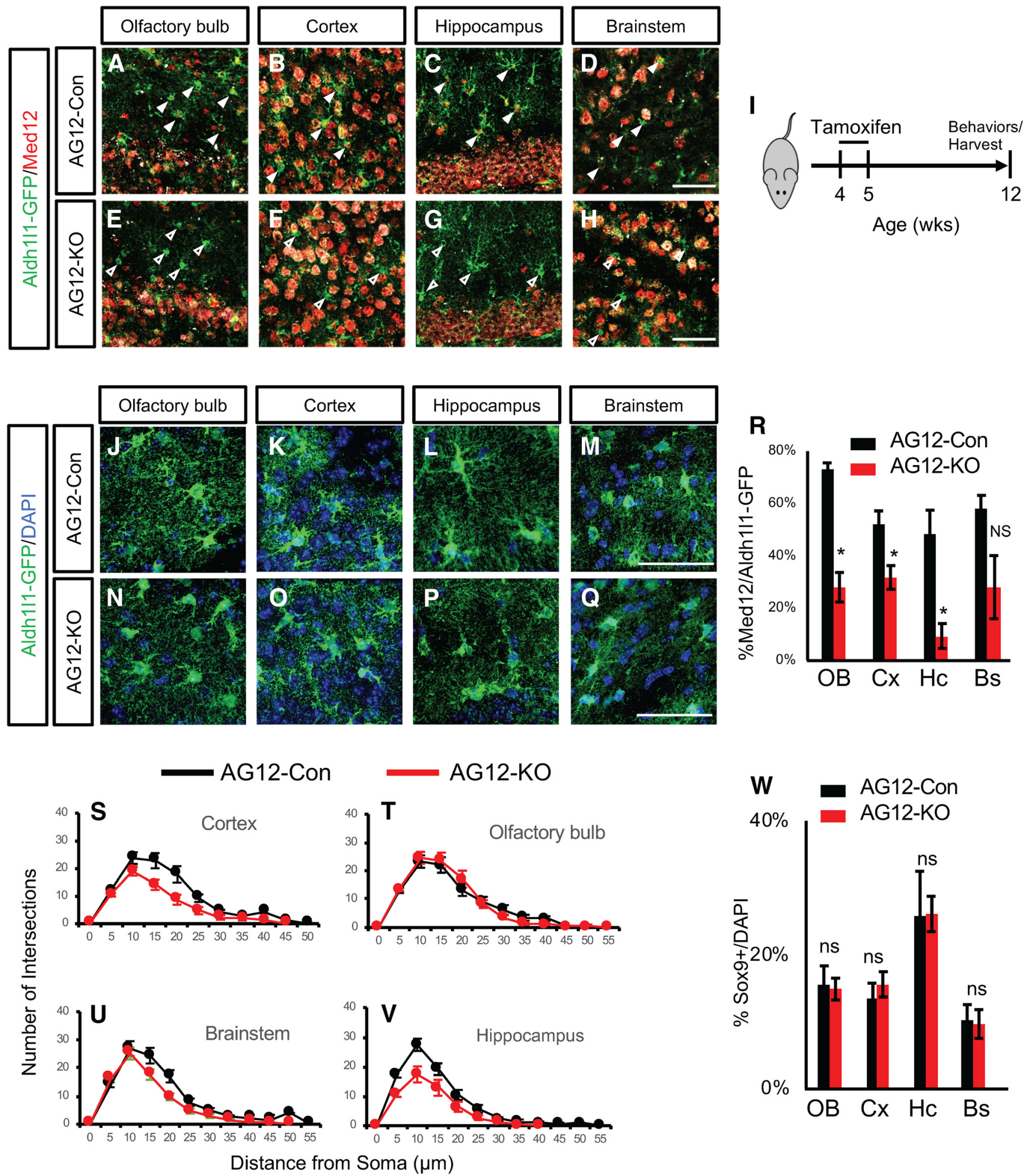

Figure 1. Deletion of Med12 in mature astrocytes selectively reduces morphologic complexity. $\boldsymbol{A}-\boldsymbol{H}$, Coexpression of Med12 and Aldh111-GFP in AG12-Con and AG12-K0 mice. Filled arrowheads show coexpression of Aldh1l1-GFP and Med12; unfilled arrowheads show Aldh111-GFP cells that do not express Med12. $I$, Tamoxifen treatment paradigm. J-Q, High-magnification confocal images of Aldh111-GFP from AG12-Con or AG12-KO mice showing reduced complexity in the cortex and hippocampus. $\boldsymbol{R}$, Quantification of Med12/Aldh1/1-GFP coexpression in AG12-Con and AG12-KO mice. Data are derived from three mice per genotype, three slides per region, per mouse: at least 500 cells per region, per mouse. One-way ANOVA. $\mathbf{S}-\boldsymbol{V}$, Scholl analysis of astrocyte complexity in the cortex, olfactory bulb, brainstem, and hippocampus. Data are derived from three mice per genotype, three slides per region, per mouse, with at least 30 cells per region per genotype; two-way repeated-measures ANOVA. $\boldsymbol{W}$, Quantification of Aldh111-GFP/DAPI coexpression in AG12-Con and AG12-KO mice. Data are derived from three mice per genotype, three slides per region, per mouse: at least 500 cells per region, per mouse. One-way ANOVA. NS: not significant. ${ }^{*} p<0.05$. 
module and color coded the reconstructed surface images based on the surface area of each astrocyte. Morphologic analysis was performed using the Imaris Filament module. Astrocyte branches and processes were outlined by Autopath with a starting point set at $10 \mathrm{~mm}$ and seed point set at $0.5 \mathrm{~mm}$, and statistical outputs including "filament number Sholl intersections" were extracted and plotted with Prism software. Data were generated from three brain sections per region per mouse, with three mice per genotype. To analyze the number of astrocytes and Med12 knockout efficiency, fluorescent images were acquired using a Zeiss LSM 880 laser-scanning confocal microscope with a $20 \times$ objective. Cell numbers were quantified by the QuPath software Cell Detection function (Bankhead et al., 2017). To examine the cochlea, fluorescent images were acquired using a Zeiss LSM 880 laser-scanning confocal microscope with a $20 \times$ objective.

\section{Behavioral tests}

Fear conditioning. Fear conditioning was performed as previously described (Samaco et al., 2008). Mice were tested at 4 months of age in a chamber that contains a grid floor that can deliver an electric shock (Actimetrics Chamber System, Med Associates). On day 1 of the test, mice were placed in the chamber and left undisturbed for $2 \mathrm{~min}$ followed by a $30 \mathrm{~s}$ white noise sound pulse ("cue"). At the end of the cue, the mouse was shocked ( $2 \mathrm{~s}, 0.4 \mathrm{~mA})$. Two minutes later, a second pairing of sound cue followed by shock was delivered. Thirty seconds after the final shock, the animal was removed and placed back to the home cage. The following day, the animals were again placed in the same chamber ("context test") and freezing behavior was recorded for $6 \mathrm{~min}$. Freezing behavior was recorded automatically by the instrument. One hour after the context test, the animals were placed into a chamber that had been cleaned with an unfamiliar agent (ethanol), and the wall color, the chamber shape, and the odor (artificial vanilla) had been changed to remove the contextual cues of the chamber. The animals were then monitored for $3 \mathrm{~min}$. After $3 \mathrm{~min}$, the white noise cue was started and lasted $3 \mathrm{~min}$. The amount of freezing was recorded separately for the first $3 \mathrm{~min}$ and for the last $3 \mathrm{~min}$ (cue test). The number of freezing intervals was converted to a percentage of freezing for both the context test and the cue test, and the data were analyzed using a one-way ANOVA.

Acoustic startle response. Acoustic startle response and prepulse inhibition were performed as previously described (Samaco et al., 2008). Mice at 4 months of age were subjected to acoustic prepulse inhibition test. The acoustic prepulse inhibition task consists of presenting the animal with two closely paired sound pulses, as follows: a prepulse at $+0 \mathrm{~dB},+4 \mathrm{~dB}(74 \mathrm{~dB}),+8 \mathrm{~dB}(78 \mathrm{~dB}),+12 \mathrm{~dB}(82 \mathrm{~dB})$, and over background followed $100 \mathrm{~ms}$ later by a pulse of $120 \mathrm{~dB}$. The amount of startle that the pulse induces in the animal is recorded using a startle chamber for mice (SR-Lab, San Diego Instruments), which records activity for $65 \mathrm{~ms}$ after the pulse. The maximum amplitude recorded over the $65 \mathrm{~ms}$ is recorded and compared using an ANOVA. No prepulse inhibition result is presented because the Med12-deficient mice showed no acoustic startle response.

Auditory brainstem response recording. Auditory brainstem response recording was performed as previously described (Manalo et al., 2020). Mice were intraperitoneally injected with ketamine/xylazine (100:10 mg/ $\mathrm{kg}$ ). The depth of anesthesia was verified by the absence of toe-pinch reflex. Testing was performed in a soundproof Faraday cage booth while mice were placed on heating pad to maintain normal body temperature throughout the procedure. Pure tone bursts $(0.1 \mathrm{~ms}$ rise/fall, $2 \mathrm{~ms}$ duration, 21 presentations/s) from 4 to $48 \mathrm{kHz}$ were generated using System 3 Digital Signal Processing hardware and software (TuckerDavis Technologies). The intensity of the tone stimuli was calibrated using a type 4938 one-quarter inch pressure-field calibration microphone (Brüel \& Kjær). EC1 ultrasonic, low-distortion electrostatic speakers were coupled to the ear canal to deliver stimuli within $3 \mathrm{~mm}$ of the tympanic membrane. Response signals were recorded with subcutaneous needle electrodes inserted at the vertex of the scalp (channel 1), the postauricular bulla region (reference), and the back leg (ground), and averaged over 500 presentations of the tone bursts. Electrode-recorded activity was filtered (high pass, $300 \mathrm{~Hz}$; low pass, $3 \mathrm{kHz}$; notch, $60 \mathrm{~Hz}$ ) before averaging to minimize background noise. Auditory thresholds were determined by decreasing the sound intensity of each stimulus to $10 \mathrm{~dB}$ from $90 \mathrm{~dB}$ in $5 \mathrm{~dB}$ steps until the lowest sound intensity with reproducible and recognizable waveforms was detected. Thresholds were determined to within $5 \mathrm{~dB}$ for each frequency by two raters to ensure reliability. SDs (dB SPL) were plotted as a function of stimulus frequency (in kilohertz) and analyzed for group differences by a two-way ANOVA, followed by a multiple-comparison test, to reveal overall trends.

\section{Distortion product otoacoustic emission}

Distortion product otoacoustic emissions (DPOAEs; 2f1-f2) were measured from the left ears in sessions separate from ABR recording. Twelve A12-KO mice ( 3 females) and 9 A12-Con mice ( 1 female) were prepared as for $\mathrm{ABR}$ measures. Input-output curves were obtained for primary stimulus frequencies $\mathrm{f} 1$ and $\mathrm{f} 2$, where $\mathrm{f} 1=\mathrm{f} 2 / 1.2$ at fixed level differences $\mathrm{L} 1$ and $\mathrm{L} 2$, where $\mathrm{L} 2=\mathrm{L} 1-10 \mathrm{~dB}$. DPOAEs were measured for $\mathrm{f} 2=12$, 18 , and $24 \mathrm{kHz}$, and $\mathrm{L} 2$ levels ranging from 0 to $80 \mathrm{~dB}$ SPL in $5 \mathrm{~dB}$ steps. Stimuli were presented using two speakers (model EC1, Tucker-Davis Technologies) configured in a closed acoustic system along with a Knowles probe microphone to record speaker and cochlear output. Primary and DPOAE levels were recorded using EMAV software in conjunction with Tucker-Davis Techologies and custom hardware. DPOAE thresholds were defined as a $2 \mathrm{f} 1-\mathrm{f} 2$ response of at least $-15 \mathrm{~dB}$ SPL. This 
A
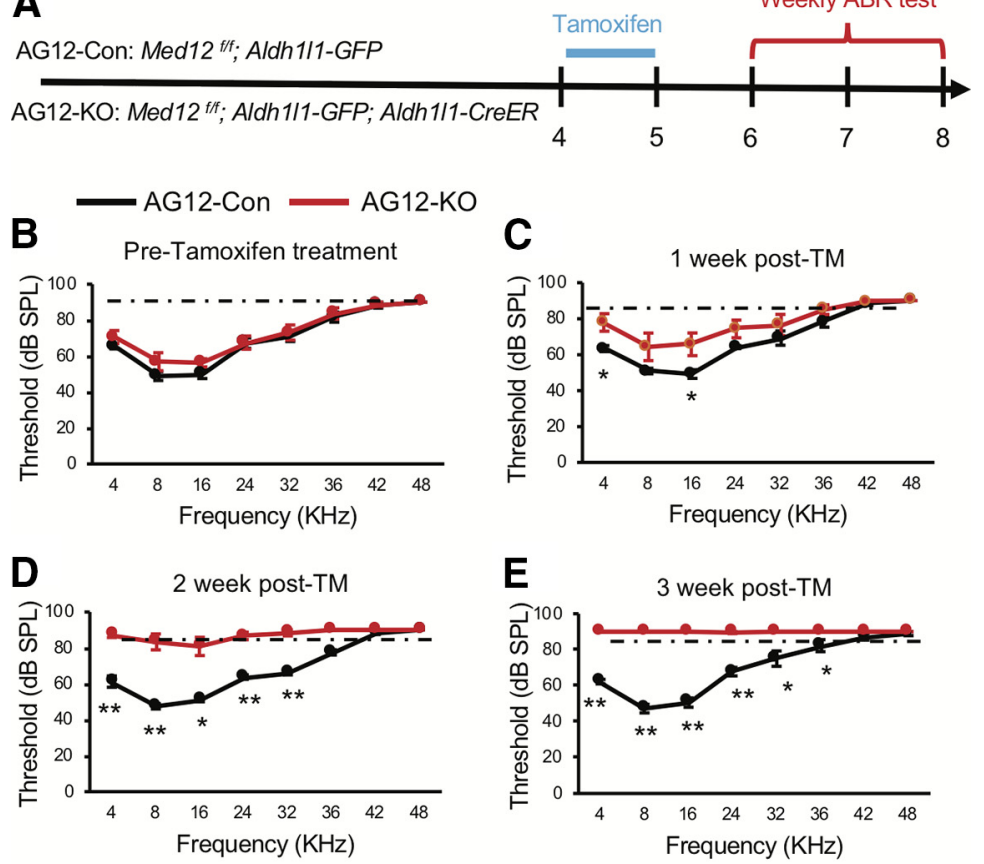

$\mathbf{F}$ Con: $8 \mathrm{KHz}, 3$ w post-TM

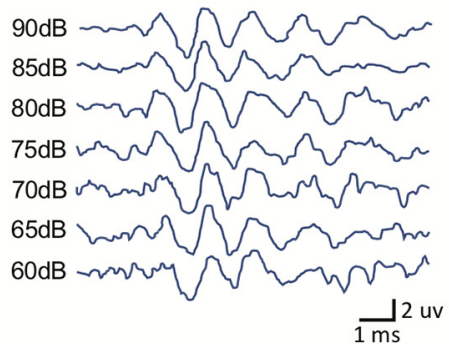

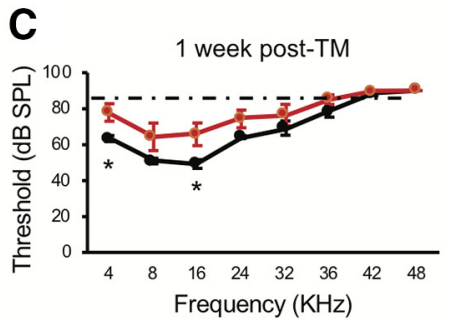

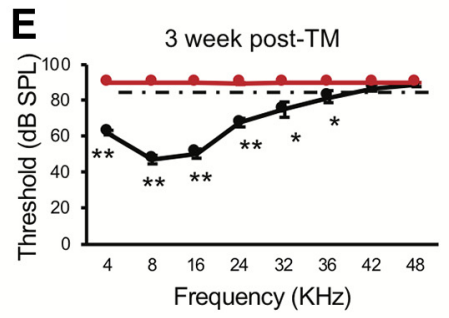

G $\quad$ cKO: $8 \mathrm{KHz}, 3 w$ post-TM

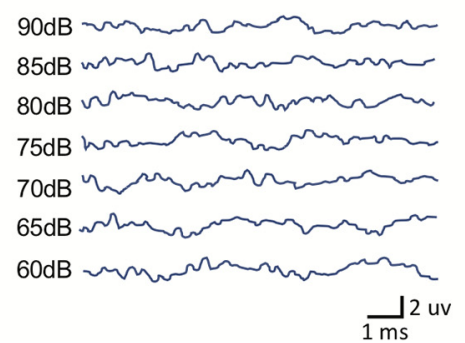

Figure 3. Med12 deletion causes rapid peripheral hearing loss in adult mice. $\boldsymbol{A}$, Tamoxifen treatment paradigm for weekly ABR tests. $\boldsymbol{B}-\boldsymbol{E}$, ABR thresholds at different frequencies before $(\boldsymbol{B})$, after 1 week $(\boldsymbol{C})$, after 2 weeks $(\boldsymbol{D})$, and after 3 weeks $(\boldsymbol{E})$ of the tamoxifen treatment. Dashed lines mark the maximum pressure, $90 \mathrm{~dB}$, used in the tests. In $\boldsymbol{B}$, Nine AG12-Con and 8 AG12-KO mice were recorded; in C, 6 AG12-Con and 7 AG12-KO mice were recorded; in D, 4 AG12-Con and 6 AG12-K0 mice were recorded; in $\boldsymbol{E}, 9$ AG12-Con and 10 AG12-K0 mice were recorded. $F, G$, ABR to stimuli of 60 $90 \mathrm{~dB}$ SPL $(8 \mathrm{kHz})$ in AG12-Con $(\boldsymbol{F})$ and AG12-KO (G) mice. AG12-Con denotes Med12//fi; Aldh111-GFP and Med12fl/y; and Aldh111-GFP. AG12-KO denotes Med12 ${ }^{\text {f/fl; }}$; Aldh111-CreER; Aldh111-GFP and Med12fl/y; Aldh111-CreER; Aldh111-GFP. Two-way repeated-measures ANOVA. ${ }^{*} p<0.05 ;{ }^{* *} p<0.01$.

level was chosen as it represented the lowest criterion response that was reliably above background noise levels and was generally within $10 \mathrm{~dB}$ of $\mathrm{ABR}$ thresholds.

\section{Endocochlear potential recording}

EP recordings were obtained from the left ear of 10 A12-KO mice ( 2 females) and 9 A12-Con mice (1 female). To record the EP, animals were anesthetized with $80 \mathrm{mg} / \mathrm{kg}$ ketamine, $15 \mathrm{mg} / \mathrm{kg}$ xylazine, i.p., and positioned ventral-side up in a custom head holder. Core temperature was maintained at $37.5 \pm 1.0^{\circ} \mathrm{C}$ using a DC electric heating pad in conjunction with a rectal probe (FHC). After blunt dissection of the jaw musculature to reveal the left auditory bulla and opening of the posterior bulla with forceps, a hole was made in the cochlear capsule directly over scala media of the lower basal turn using a fine drill. Glass capillary pipettes (20-30 M $\Omega$ ) filled with $0.15 \mathrm{M} \mathrm{KCl}$ were mounted on a hydraulic microdrive (Frederick Haer) and advanced until a stable positive potential was observed that did not change with increased electrode depth. The signal from the recording electrode was led to an intracellular amplifier (Model 1600, AM Systems).

Quantification and statistical analysis

Sample sizes and statistical tests can be found in accompanying figure legends. Offline analysis was conducted using SPSS 20 and Excel

software. We assessed the significance of data for comparison by Student's two-tailed unpaired/ paired $t$ test or two-tailed unpaired $t$ test. For multiple comparisons, we used the one-way ANOVA with Tukey's test and two-way ANOVA with Sidak's test. In general, we assumed that data were normally distributed, but this was not formally tested. Data are presented as the mean \pm SEM. Levels of statistical significance are indicated as follows: ${ }^{*} p<0.05,{ }^{* *} p<0.01,{ }^{* *} p<0.001$.

\section{Results}

Deletion of Med12 selectively reduces morphologic complexity of astrocytes To decipher whether Med12 plays a role in astrocyte function in the adult brain, we evaluated its expression in astrocytes by coimmunostaining with GFP and Med12 in 16-weekold Aldh1l1-GFP reporter mice. This analysis revealed that $\sim 70 \%$ of Aldh1l1-GFP astrocytes express Med12 in the olfactory bulb (73.1 \pm $2.3 \%)$ and $\sim 50 \%$ across other examined brain regions (Fig. $1 A-D, R$; cortex, $52.0 \pm 2.3 \%$; hippocampus, $48.5 \pm 8.8 \%$; brainstem, $57.9 \pm$ $5.2 \%)$; Med12 also exhibited expression in NeuN-positive neurons in these same brain regions.

Med12 plays an important role in numerous developing tissues, rendering the germline embryonic knockout lethal (Rocha et al., $2010 a, b)$ and requiring the generation of temporal and lineage-specific knock-out alleles to study its role in mature astrocytes. To specifically knock out Med12 in astrocytes in the adult brain, we acquired mice containing a floxed Med12 allele $\left(\operatorname{Med} 12^{f l / f l}\right)$ and intercrossed it with the Aldh1l1-CreER line and the Aldh1l1-GFP reporter, producing the Aldh1l1-CreER; Aldh1l1-GFP; Med12 fllfl and Aldh1l1CreER; Aldh1l1-GFP; Med12 fl/y (AG12) mouse lines. TM was administered at postnatal day 30 (P30), which allowed us to bypass possible developmental roles for Med12. Mice were harvested 2 months after TM treatment (P80 to P90) and stained for Med12. We observed the loss of Med12 in $>50 \%$ of Aldh1l1-GFP-positive astrocytes in the olfactory bulb, cortex, hippocampus, and brainstem 2 months after treatment (Fig. $1 A-H, R$; in Aldh1l1-GFP astrocytes that express Med12 in AG12-Con: olfactory bulb, $73.1 \pm 2.3 \%$; cortex, $52.0 \pm 5.1 \%$; hippocampus, $48.5 \pm 8.8 \%$; brainstem, $57.9 \pm 5.2 \%$; in AG12-KO: olfactory bulb, $28.0 \pm 5.6 \%$; cortex, $31.7 \pm 4.5 \%$; hippocampus, $9.4 \pm 4.7 \%$; brainstem, $28 \pm 12 \%)$. We were unable to achieve higher $\mathrm{KO}$ efficiency because prolonging TM treatment led to severe gastrointestinal illness, which ultimately resulted in death.

To determine whether the deletion of Med12 impacts astrocyte homeostasis, we assessed their morphology using confocal imaging of the Aldh111-GFP reporter in AG12-Con and AG12KO brains. Three-dimensional reconstructions of these images were subjected to Sholl analysis to evaluate the morphologic complexity of astrocytes. This analysis revealed that astrocytes 
from AG12-KO mice demonstrate reduced complexity in the prefrontal cortex and CA1 of the hippocampus, whereas astrocytes from the olfactory bulb and brainstem were unaffected (Fig. 1J-Q,S-V). Quantification of the gross numbers of Sox9-expressing astrocytes did not reveal any differences between AG12-Con and AG12-KO mice (Aldh1l1-GFP ${ }^{+} / \mathrm{DAPI}$ ratio in AG12-Con: olfactory bulb, $15.5 \pm 2.8 \%$; cortex, $13.6 \pm 2.2 \%$; hippocampus, $25.7 \pm$ $6.8 \%$; brainstem, $10.3 \pm 2.3 \%$; in $\mathrm{AG} 12$ $\mathrm{KO}$ : olfactory bulb, $14.9 \pm 1.6 \%$; cortex, $15.6 \pm 1.9 \%$; hippocampus, $26.1 \pm 2.7 \%$; brainstem, $9.6 \pm 2.2 \%$ ), indicating that loss of Med12 does not lead to cell death (Fig. $1 W)$. Together, these studies indicate that Med12 is selectively required to maintain the morphologic complexity of astrocytes in the hippocampus and cortex, but is not required in the olfactory bulb or brainstem.

\section{Elimination of Med12 leads to rapid hearing loss}

The foregoing observation that the loss of Med12 results in decreased morphologic complexity of astrocytes in the hippocampus and prefrontal cortex, led us to examine whether these cellular phenotypes lead to behavioral deficits associated with these brain regions. Since the hippocampus demonstrated the most efficient knockout of Med12, we conducted fear-conditioning assays, which are associated with hippocampal function. As shown in Figure 2A, AG12-Con mice showed significantly increased freezing behaviors after the auditory cue (AG12Con mice: no cue, $25.3 \pm 1.5 \%$; cued, $75.2 \pm 3.2 \%$ ); however, AG12-KO mice demonstrated no change in freezing before and during the auditory cue (Fig. $2 A$; AG12-KO mice: no cue, $6.7 \pm 1.7 \%$; cued, $17.1 \pm 5.4 \%)$. Further analysis revealed that the AG12-KO mice did not respond to acoustic stimulation (Fig. $2 B$; AG12-Con mice, $2495.2 \pm 492.1$ a.u.; AG12-KO mice, $58.0 \pm 11.9$ a.u.), indicating that the apparent deficits in fear conditioning are likely the result of impaired hearing. Consistent with this interpretation, we did not observe any changes in short-term potentiation (STP) or long-term potentiation (LTP) in the hippocampus of AG12-KO mice compared with AG12Con mice (Fig. 2C-E; LTP: AG12-Con mice, $120.69 \pm 14.43 \%$; AG12-KO mice, $124.8 \pm 25.67 \%)$.

The loss of hearing in the AG12-KO mice prompted us to further examine auditory function by measuring ABRs. We first established a baseline ABR for AG12-Con and AG12KO mice before TM-induced deletion of Med12, finding no differences in ABR thresholds between two groups (Fig. 3B; $4-32 \mathrm{kHz}$ ). Next, we evaluated ABR in weekly intervals post-
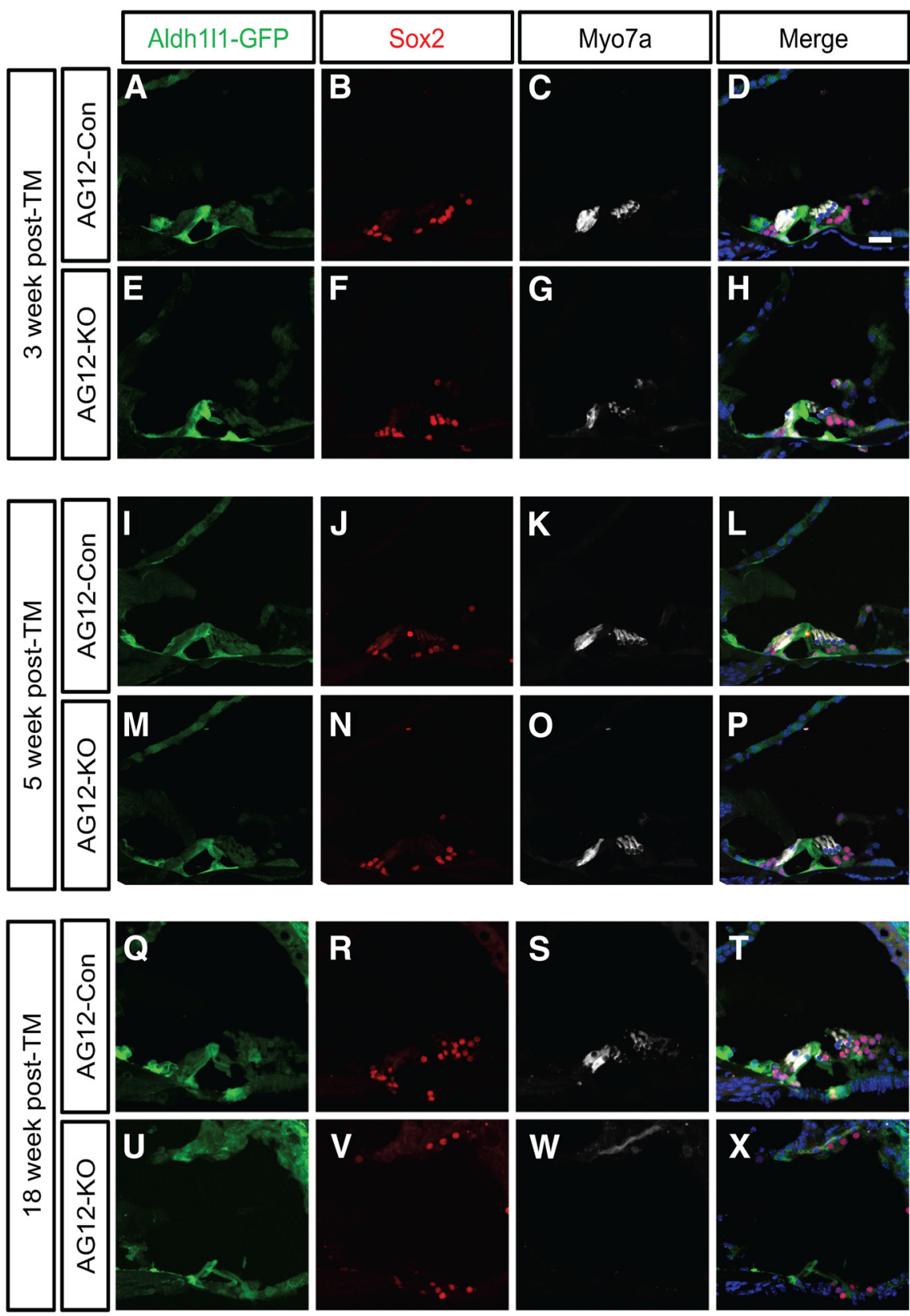

Figure 4. Deletion of Med12 has latent effects on the structure of organ of Corti. $\boldsymbol{A}-\boldsymbol{X}$, Detection of the markers of supporting cells, Sox2, and hair cells, My07a, by immunofluorescence on midmodiolar sections of cochleae of AG12-Con and AG12-KO mice. Figures show the organ of Corti in the basal turn. A comparison of midmodiolar sections of cochleae of AG12-Con and AG12-K0 mice 3 weeks post-TM $(\boldsymbol{A}-\boldsymbol{H})$, 5 weeks post-TM $(\boldsymbol{I}-\boldsymbol{P})$, and 18 weeks post-TM (Q-X). AG12-Con denotes Med12 ${ }^{\mathrm{fl} / \mathrm{fl}}$; Aldh1l1-GFP and Med12fl/y; Aldh1l1-GFP. AG12-K0 denotes Med12 fl/fi; Aldh1l1-CreER; Aldh1l1-GFP and Med12fl/y; Aldh1l1CreER; Aldh111-GFP. Scale bar, D, $20 \mu \mathrm{m}$.

TM-induced deletion (Fig. 3A), finding dramatic elevation of auditory thresholds at 1 week post-TM treatment (at 4 $\mathrm{kHz}$ : AG12-Con mice, $63.3 \pm 1.7 \mathrm{~dB}$ SPL; AG12-KO mice, $77.9 \pm 4.9 \mathrm{~dB}$ SPL; at $16 \mathrm{kHz}$ : AG12-Con mice, $49.2 \pm 2.4 \mathrm{~dB}$ SPL; AG12-KO mice, $65.7 \pm 6.3 \mathrm{~dB}$ SPL), culminating in a complete loss of auditory response to all stimulation by 3 weeks post-TM treatment (Fig. 3C,D; 4-32 kHz; AG12-KO mice with no response). To identify the possible cause of hearing loss, we further examined the recording of auditory brainstem responses from each group at 3 weeks after TM induction. $\mathrm{ABR}$ responses at $8 \mathrm{kHz}$ in $\mathrm{AG} 12-\mathrm{Con}$ mice revealed five clearly defined maxima, which represent major ABR peaks I to $\mathrm{V}$ (Fig. $3 F$ ). In contrast, we did not observe 

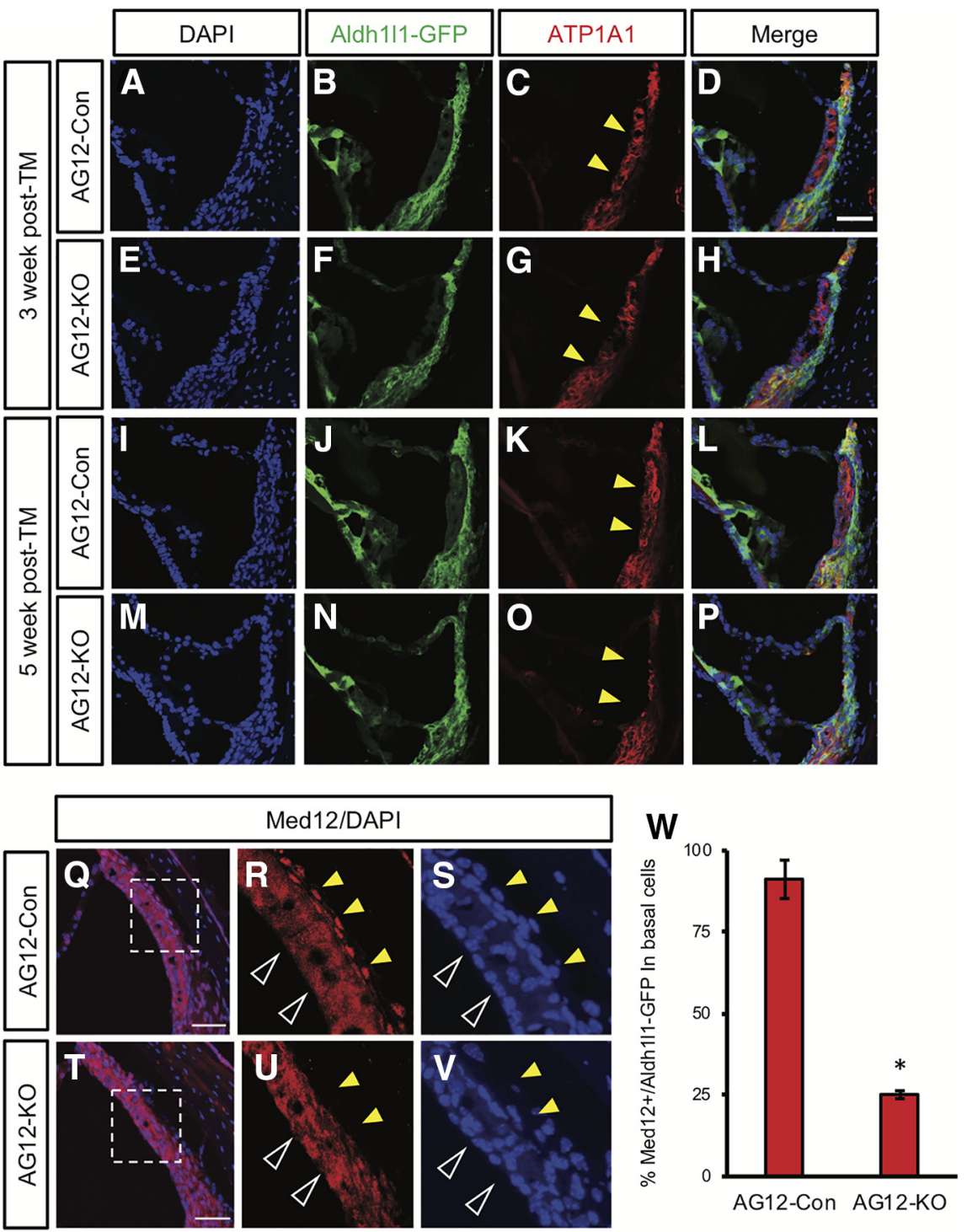

Figure 5. Med12 expression in basal cells is required to maintain the stria vascularis. $\boldsymbol{A}-\boldsymbol{P}$, Detection of the stria vascularis marker, ATP1A1, by immunofluorescence on midmodiolar sections of cochleae of AG12-Con and AG12-K0 mice. Figures show the stria vascularis in the apical turn. Yellow arrowheads mark the regions of stria vascularis. A comparison of midmodiolar sections of cochleae of AG12-Con and AG12-K0 mice 3 weeks post-TM $(\boldsymbol{A}-\boldsymbol{H})$ and 5 weeks post-TM (I-P). Q-V, Immunostaining of Med12 in stria vascularis of midmodiolar sections of cochleae of AG12-Con and AG12-KO mice at 2 weeks post-TM. Figures show the stria vascularis in the middle turn. $\boldsymbol{R}-\boldsymbol{S}$ and $\boldsymbol{U}-\boldsymbol{V}$ are the regions from dashed squares in $\mathbf{Q}$ and $\boldsymbol{T}$. Unfilled arrowheads mark the sides of intermediate cells; yellow arrowheads mark the sides of basal cells and the spiral ligament. AG12-Con denotes Med12 fl/fl; Aldh1l1-GFP and Med12fl/y; Aldh1l1-GFP. AG12-KO denotes Med12 fl/fl; Aldh1l1-CreER; Aldh1l1-GFP and Med12fl/y; Aldh1l1-CreER; Aldh1l1-GFP. W, Quantification of Med12 expression in Aldh1l1-GFP basal cells; ${ }^{*} p<0.05$. Scale bars: $\boldsymbol{D}, 50 \mu \mathrm{m} ; \mathbf{Q}, 20 \mu \mathrm{m}$.

any of the major ABR peaks in AG12-KO mice 3 weeks after TM treatment (Fig. 3G). ABR wave I corresponds to compound auditory nerve conduction; therefore, its loss in the AG12-KO mice indicates that Med12 deletion results in dysfunction of the inner ear, which inhibits initiation of the auditory response.

Med12 is required to maintain the structural integrity of the stria vascularis

Having established that the loss of hearing in AG12-KO mice is likely because of defects in the inner ear, we next examined the cellular integrity of the cochlea. Starting with the organ of Corti, analysis of AG12-KO mice at 3 weeks post-TM induction did not reveal any overt structural changes in supporting cells $\left(\mathrm{Sox} 2^{+}\right)$or hair cell $\left(\right.$ Myo7a $^{+}$) populations (Fig. $4 A-H$; three animals in each group were analyzed). Moreover, analysis at 5 weeks post-TM induction also did not show any changes in the cellular integrity of the organ of Corti (Fig. 4I-P; three animals in each group were analyzed). However, analysis at 18 weeks post-TM induction did reveal an eventual loss of Myo7a-expressing hair cells, coupled with disorganization of Sox2-expressing support cells and a general breakdown in organ of Corti integrity (Fig. 4Q-X; three animals in each group were analyzed). Nevertheless, the latent loss of hair cells at 18 weeks after Med12 deletion does not coincide with the observed loss of ABR at 2-3 weeks postdeletion (Fig. 3), indicating that Med12-dependent loss of ABR is likely mediated through other cellular mechanisms in the adult cochlea.

Next, we examined additional structures in the cochlea, finding that Aldh1l1-GFP is also colocalized to the spiral ligament and basal cells of the stria vascularis (Fig. $5 A-D$ ). Strikingly, at 3 weeks post-TM induction the stria vascularis of AG12-KO mice exhibited drastic alterations in its integrity, highlighted by alterations in the structure of ATP1A1-expressing intermediate cells at the apical turn (Fig. 5, compare $C, D$, $G, H$, yellow arrows). Analysis at 5 weeks post-TM revealed that ATP1A1-expressing intermediate cells are completely lost from middle to apical turns in AG12-KO mice, which further disrupts the architecture of these structures (Fig. 5I-P). Given the essential role of the stria vascularis in maintaining the ionic composition of endolymph, these findings suggest that progressive loss of the stria vascularis results in ionic imbalance of the endolymph, which compromises hair cell function, leading to hearing loss in AG12-KO mice.

To decipher which cell populations in the cochlea are dependent on Med12 expression, we used immunostaining to determine its pattern of expression. Staining for Med12 was observed in the stria vascularis in AG12-con mice 3 weeks post-TM induction (Fig. 5Q,R). Nuclear localization of Med12 was observed in basal cells and fibrocytes in the spiral ligament (Fig. $5 R$, yellow arrowheads). Analysis of Med12 expression in AG12-KO mice revealed that the nuclear localized expression in basal cells was significantly decreased (Fig. 5 , compare $R, T$ ). The percentage of Med12-positive basal cells reduced from $91.2 \pm 5.9 \%$ (AG12-con mice, $n=3$ ) to $25.1 \pm 1.2 \%$ (AG12KO mice, $n=3$; Fig. $5 W$ ). These observations suggest that Med12 deletion is occurring in the basal cells of the stria vascularis in the AG12-KO mouse line. 


\section{Med12 deletion disrupts basal cell organization}

To track which populations in the cochlea undergo TM-induced Crerecombination in the Aldh1l1-CreER line, we replaced the Aldh1l1-GFP reporter with a Cre-inducible reporter, generating the Aldh1l1-CreER; RosaCAG-LSL-tdTomato; Med12 $2^{f l / f}$ mouse line (i.e., AT12 line). We treated AT12Con mice with TM and harvested the cochlea 2 weeks after induction, finding that the tdTomato reporter labeled the Glut1-expressing basal cells of the stria vascularis and some fibrocytes in the spiral ligament (Fig. $6 A-D$, yellow arrowheads), but not spiral ganglion cells (data not shown). Consistent with a requirement for Med12 expression in basal cells, examination of AT12-KO mice 2 weeks after TM induction revealed a loss of basal cell organization, where Glut1- and tdTomatoexpressing basal cells appear diffuse and lose cell-cell contact (Fig. 6E-H, unfilled arrowheads; three animals in each group were analyzed, phenotype was observed in all AT12-KO mice). Together, these findings indicate that Med12 is required to maintain the organization and integrity of basal cells in the stria vacularis.

Adhesion between cell types within the stria vascularis is required to generate and preserve ion gradients in the endolymph, which are essential for maintaining endocochlear potential and auditory function (Ciuman, 2009; Wan et al., 2013). Our observed cellular phenotypes in basal cells of the AT12-KO line suggest that cell adhesion within the stria vascularis is disrupted. To test this, we evaluated the expression of key junction proteins associated with hearing loss in this region, $\mathrm{ZO} 1, \mathrm{Ecad}$, and $\mathrm{Cx} 31$. In AT12-Con animals, ZO1 is concentrated next to the cell membrane of basal cells; however, ZO1 expression level was dramatically decreased in AT12-KO mice (Fig. 6I-L, white arrowheads; the intensity ratio was reduced to $0.696 \pm 0.026$ compared with AT12-Con; $n=3$ in each group). Cx31 protein was highly concentrated between basal cells and fibrocytes in spiral ligament in AT12-Con mice and demonstrated disorganized localization in AT12-KO mice (Fig. 6M-P, white arrowheads; Fig. $6 U$, the intensity ratio was reduced to $0.689 \pm 0.103$ compared with AT12-Con; $n=3$ in each group). Ecad expression was mainly observed in both intermediate cells and basal cells (Fig. 6Q, $R)$. Although the distribution of Ecad was altered in AT12-KO mice (Fig. 6S,T), the intensity was not significantly different compared Scale bars: $\boldsymbol{D}, \boldsymbol{L}, 50 \mu \mathrm{m}$.
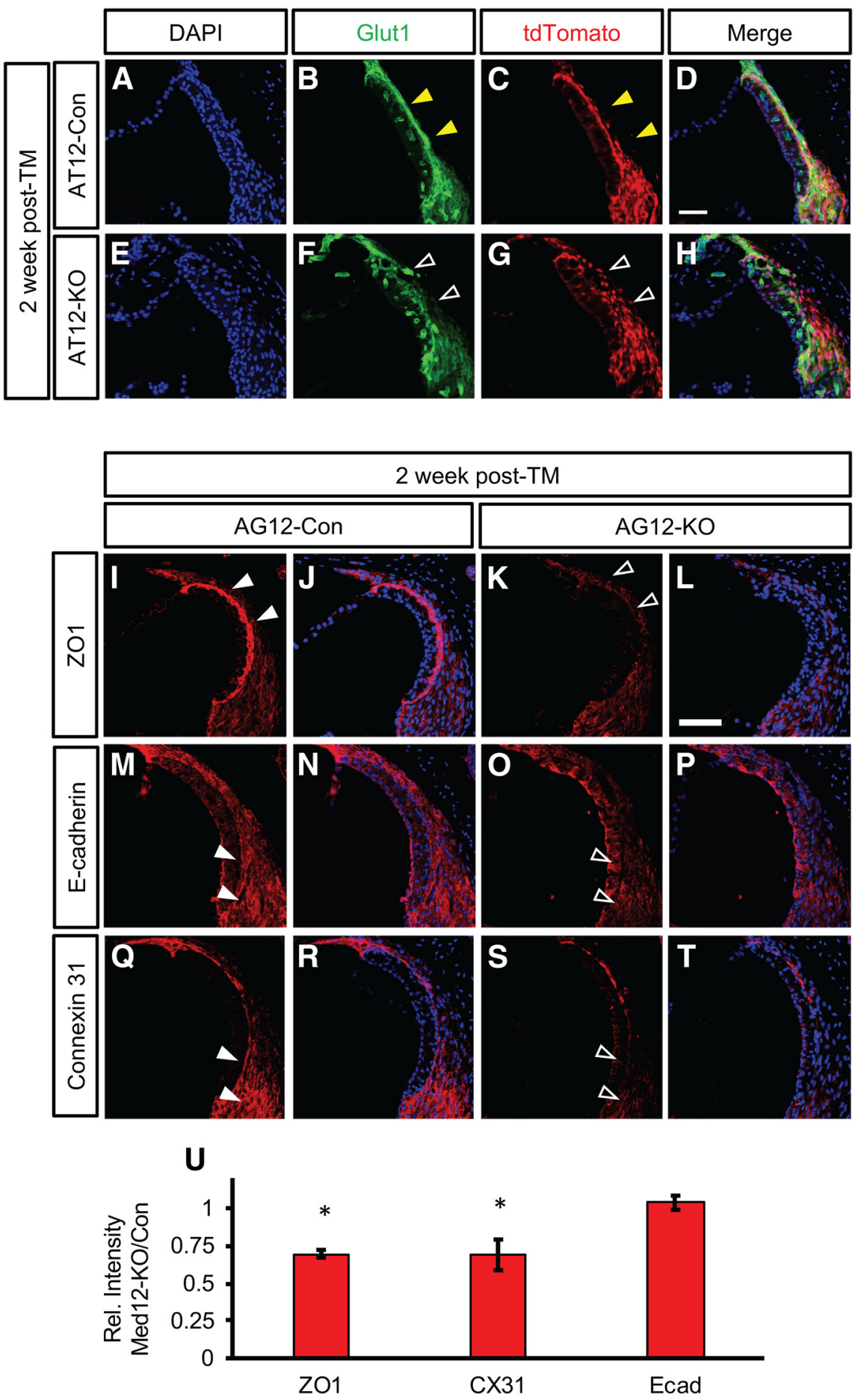

Figure 6. Med12 is required to maintain expression of cell adhesion proteins. $\boldsymbol{A}-\boldsymbol{H}$, Detection of Glut1, an endothelial and basal cell marker, by immunofluorescence on midmodiolar sections of cochleae of AT12-Con and AT12-KO mice at 2 weeks post-TM. Figures show the stria vascularis in the middle turn. Yellow arrowheads denote Glut1-positive basal cells, labeled by AT12-Con tdTomato reporter $(\boldsymbol{B}, \mathbf{C})$; unfilled arrowheads denote disorganized Glut-1/tdTomato basal cells in AT12-KO $(\boldsymbol{F}, \boldsymbol{G})$. $\boldsymbol{I}-\boldsymbol{T}$, Detection of Z01, E-cadherin, and Connexin-31 proteins by immunofluorescence on midmodiolar sections of cochleae of AG12-Con and AG12-KO mice at 2 weeks post-TM. I, $\boldsymbol{M}, \mathbf{Q}$, Filled arrowheads denote AG12-Con expression of makers. $\boldsymbol{K}, \mathbf{0}, \mathbf{S}$, Unfilled arrows denote altered expression of markers in AG12-K0. $\boldsymbol{U}$, Quantification of immunostaining from I to $T^{*}{ }^{*} p<0.05$.

with AT12-Con mice (Fig. 6Q-T,U; intensity ratio, $1.036 \pm 0.047$; compared with AT12-Con; $n=3$ in each group). These findings suggested that Med12 is necessary to maintain the expression of these critical junction and cell adhesion proteins in the stria vascularis and spiral ligament. 

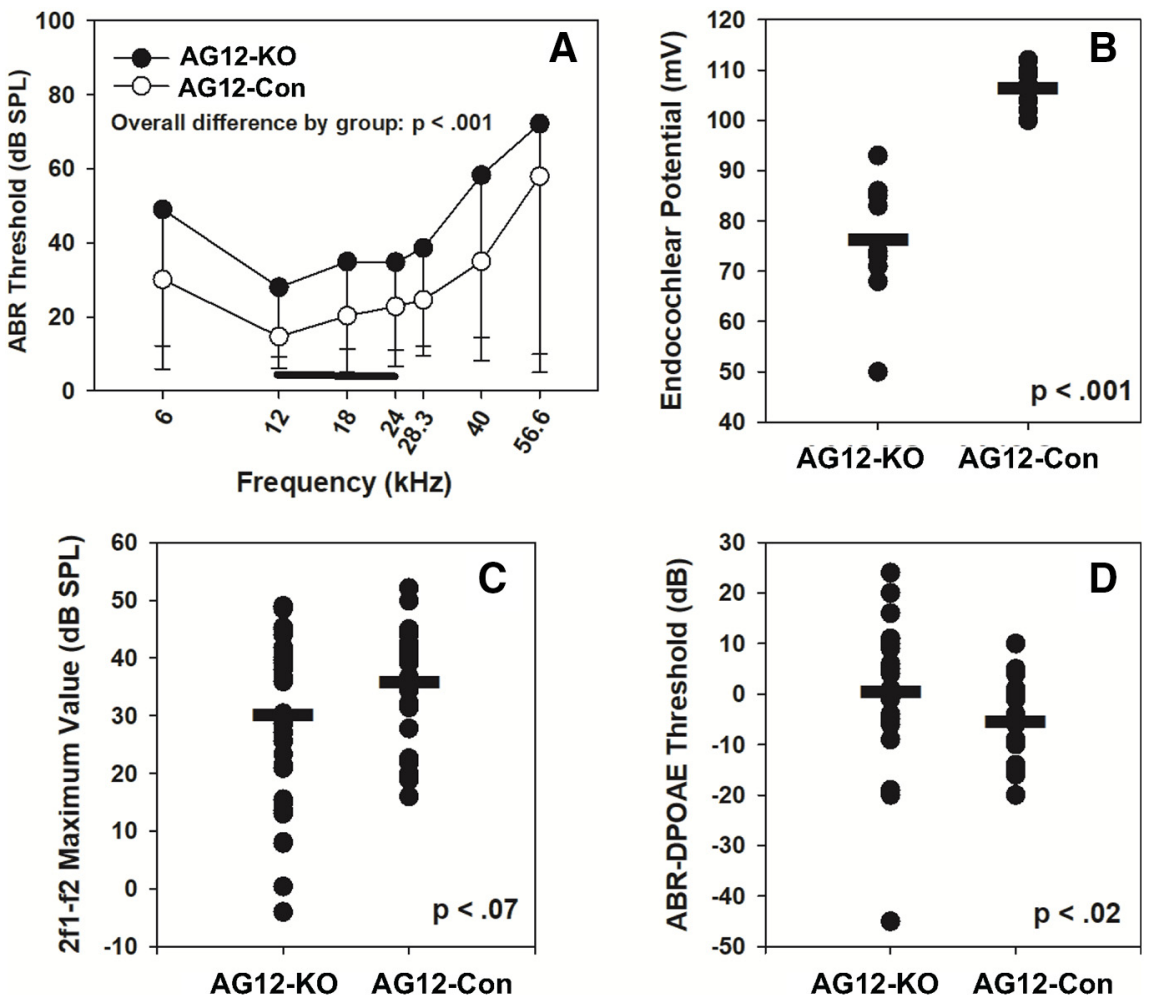

Figure 7. Loss of Med12 results in stria vascularis-specific defects. $A, A B R$ thresholds were significantly higher in KOs versus controls (two-way ANOVA, $F=46.188, \mathrm{df}=6, p<0.001$ ) with no significant interactions. $\boldsymbol{B}$, EPs were significantly lower in KOs ( $t$ test for unequal variance, $\mathrm{df}=11, t=-7.23, p<0.001$ ). C, 2f1-f2 maximum values were not significantly different by group ( $t$ test for unequal variance, $\mathrm{df}=60, t=-1.84, p<0.07$ ). $\boldsymbol{D}$, Difference in ABR versus DPOAE threshold (ABR-DPOAE for $-15 \mathrm{~dB}$ SPL criterion) was significantly higher in KOs ( $t$ test for unequal variance, $\mathrm{df}=59, t=2.32$, $p<0.02$ ). DPOAE metrics were derived from input-output curves for $\mathrm{f} 2=12,18$, and $24 \mathrm{kHz}$ (see horizontal bar in $\boldsymbol{A}$ ). Since these curves did not differ by $\mathrm{f} 2$ (data not shown), data from all three frequencies were included in $\boldsymbol{C}$ and $\boldsymbol{D}$.

\section{Reduced endocochlear potential in Med12 mutant mice} The foregoing studies indicate that the loss of Med12 disrupts the organization of the basal cells as early as 2 weeks after TMinduced deletion, suggesting that the cascade of cellular events that lead to hearing loss originates in the stria vascularis. Therefore, we next measured the EP in AG12-Con and AG12KO mice 10-14 d after TM-induced deletion. Consistent with our prior results (Fig. 3), AG12-KO mice showed ABR thresholds that were $15-25 \mathrm{~dB}$ higher than those in AA2-Con mice. These differences were significant overall $(p<0.001)$, with no interactions by frequency (Fig. $7 A$ ). The overall relative "flat" threshold shift in the AG12-KO mice compared with AG12-Con supports a non-frequency-specific influence, consistent with isolated strial dysfunction (Schuknecht and Gacek,1993). EP recordings from the same cochleas (Fig. 7B) revealed no overlap of EP values by group $(p<0.001)$, with AG12-Con mice showing typical values for C57BL/6 mice (>100 mV; Ohlemiller, 2009) and AG12-KO mice averaging $\sim 30 \mathrm{mV}$ lower (76.8 vs $106.7 \mathrm{mV}$ ).

To confirm that these mice, at this time point, have stria-specific defects, we next performed distortion DPOAE tests on these same mice (Ueberfuhr, et al., 2016). In the presence of an isolated strial defect, maximum DPOAE values should be similar for normal and stria-impaired mice, and DPOAE thresholds should be spared relative to ABR thresholds at the same frequencies (Mills, 2003). Since the DPOAE input/output results did not vary with $\mathrm{f} 2$ frequency, data from $\mathrm{f} 2=12,18,24 \mathrm{kHz}$ were all included from each animal. This analysis revealed that the maximum
DPOAE values did not differ significantly between AG12-Con and AG12-KO mice, although control mice gave slightly higher values (30.5 vs $36.2 \mathrm{~dB}$ SPL; Fig. $7 C$ ). Finally, a stria-specific defect is also predicted to yield DPOAE thresholds that are lower relative to ABR thresholds, meaning that ABR-DPOAE threshold differences should be higher when the stria is compromised. Indeed, this metric both averaged $(0.83$ vs $-5.04 \mathrm{~dB})$ and skewed higher in the AG12-KO mice (Fig. 7D), supporting the existence of isolated strial dysfunction in the majority of AG12-KO mice. In sum, $\mathrm{ABR}, \mathrm{EP}$, and DPOAE data supported a central tendency toward isolated strial dysfunction in the AG12-KO mice as early as 10-14 d after TM-induced deletion.

\section{Discussion}

Mutations in MED12 are linked to a broad spectrum of genetic disorders with $\mathrm{X}$ linked intellectual disability (Schwartz et al., 2007; Lesca et al., 2013; Vulto-van Silfhout et al., 2013; Donnio et al., 2017; Rubinato et al., 2020); however, embryonic lethality in Med12-null and heterozygous mice have hindered the study of these Med12-associated phenotypes (Rocha et al., 2010a,b). Here, we applied temporal and tissue-specific approaches to study Med12 function in adult astrocytes in the CNS. Critically, the lethality rate dramatically increased with prolonged tamoxifen treatment, which likely prevented us from achieving a high $\mathrm{KO}$ efficiency in astrocytes in the CNS (Fig. 1R). This technical limitation may have masked some CNS-related phenotypes associated with Med12 function. Surprisingly, we found that Aldh1l1-CreER-mediated Med12 deletion in the adult led to rapid hearing loss. Although hearing loss has been reported in cases of Med12-related disorders (Rubinato et al., 2020), the underlying mechanisms remained undefined. We found that Med12 is necessary for maintaining the integrity of the stria vascularis in the adult, likely by regulating the expression of $\mathrm{ZO}-1$, Ecad, and $\mathrm{Cx} 31$. These changes in gene expression alter cell adhesion between basal cells and the spiral ligament, which would likely impede the generation of normal endolymph and culminates in loss of hair cell mechanotransduction.

Our studies revealed that basal cells and the spiral ligament of the adult cochlea are transcriptionally dependent on Med12 to maintain their overall structural integrity. We recently identified similar transcriptional dependencies in the adult brain, where the transcription factor NFIA is required to maintain the morphologic complexity of hippocampal astrocytes (Huang et al., 2020). Together, these studies highlight adult- and region-specific roles for developmental transcription factors in maintaining key aspects of cell structure in mature populations. Med12 functions, in part, to maintain the expression of key proteins that maintain tight junctions (TJs) and gap junctions (GJs), which are critical for normal cochlear function (Wangemann, 2006; Wan et al., 2013). Prior studies indicate that genetic deletion of either 
Ecad (Trowe et al., 2011) or Cx31 (López-Bigas et al., 2001) during development results in hearing loss, suggesting that their regulation by Med12 contributes to the observed hearing loss in the adult. While our studies suggest that the downregulation of these structural proteins is responsible for the effects of Med12 loss in the cochlea, it is possible that Med12 regulates the expression of other genes that are also important for these effects. Further studies on Med12 target gene networks in basal cells will be critical for deciphering these regulatory networks and associated mechanisms.

Our findings demonstrate that the loss of Med12 in basal cells and that the spiral ligament leads to defects of the stria vascularis. Progressive degeneration of the stria vascularis underlies a form of age-related hearing loss known as strial or metabolic presbycusis (Schuknecht and Gacek, 1993). However, we cannot exclude the possibility that the loss of Med12 in other tissues contributes to hearing loss in humans. Med12 expression is also observed in supporting cells in cochlea and in major cell types in nervous system, raising the possibility that it has related functions in these systems. Nevertheless, our study describes the first animal model that recapitulates hearing loss identified in Med12related disorders and provides a new system in which to examine the underlying cellular and molecular mechanisms. Moreover, the TJ and GJ proteins that are downregulated in the Med12-deficient cochlea also play important roles in other systems; for example, ZO1 is highly expressed in circumventricular organs of the CNS and is required for the integrity of blood-brain barrier (Petrov et al., 1994), and a Cx31 mutation leads to peripheral neuropathy in addition to sensorineural hearing impairment in patients (López-Bigas et al., 2001). Therefore, our studies lay the foundation for further examination of Med12 function in other systems linked to Med12-disorders.

To ascertain whether the initial defects in the AG12-KO cochlea are because of strial dysfunction, we measured a series of physiological parameters associated with cochlear function at time points where basal cell organization is altered and the organ of Corti is intact. The most essential evidence for strial pathology in the AG12-KO mouse derives from direct EP recordings, where AG12-KO and AG12-Con mice showed no overlap of EP values, with the AG12-KO mice averaging an $\sim 30 \mathrm{mV}$ decrease in EP. Thus, all of the AG12-KO mice exhibited the single functional requirement for strial pathology, although this does not directly address whether other defects are present at these relatively early time points (i.e., 10-14 d post-TM). Other evidence for strialspecific defects at these early time points are based on no changes in the DPOAE input/output data between the AG12-KO and AG12-Con mice, coupled with a relative sparing of DPOAE thresholds versus ABR (DPOAE-ABR) thresholds at the same frequency (Mills, 2003), which yielded higher positive values (ABR threshold higher) in the AG12-KO line. In sum, four functional metrics supported an initial, delimited, strial pathology in AA12-KO mice. EP reduction was the requisite criterion, but since EP recording is a terminal procedure, it cannot be used clinically. While we did not first conceive of the threshold and DPOAE-based metrics, we used to evaluate strial pathology in our AG12-KO mice, we believe we are the first to apply all three criteria. The principal limitation to such an approach at present is simply the lack of suitable models. Thus, the Med12 KO model may represent a useful system for additional studies aimed at detecting and characterizing isolated chronic EP reduction.

\section{References}

Allen NJ, Lyons DA (2018) Glia as architects of central nervous system formation and function. Science 362:181-185.

Bankhead P, Loughrey MB, Fernández JA, Dombrowski Y, McArt DG, Dunne PD, McQuaid S, Gray RT, Murray LJ, Coleman HG, James JA, Salto-Tellez M, Hamilton PW (2017) QuPath: Open source software for digital pathology image analysis. Sci Rep 7:16878.

Boettger T, Hübner CA, Maier H, Rust MB, Beck FX, Jentsch TJ (2002) Deafness and renal tubular acidosis in mice lacking the K-Cl co-transporter Kcc4. Nature 416:874-878.

Brownell WE, Bader CR, Bertrand D, de Ribaupierre Y (1985) Evoked mechanical responses of isolated cochlear outer hair cells. Science 227:194196.

Chan DK, Hudspeth AJ (2005) Mechanical responses of the organ of corti to acoustic and electrical stimulation in vitro. Biophys J 89:4382-4395.

Chen J, Zhao HB (2014) The role of an inwardly rectifying $\mathrm{K}(+)$ channel (Kir4.1) in the inner ear and hearing loss. Neuroscience 265:137-146.

Ciuman RR (2009) Stria vascularis and vestibular dark cells: characterisation of main structures responsible for inner-ear homeostasis, and their pathophysiological relations. J Laryngol Otol 123:151-162.

Donnio L-M, Bidon B, Hashimoto S, May M, Epanchintsev A, Ryan C, Allen W, Hackett A, Gecz J, Skinner C, Stevenson RE, de Brouwer APM, Coutton C, Francannet C, Jouk P-S, Schwartz CE, Egly J-M (2017) MED12-related XLID disorders are dose-dependent of immediate early genes (IEGs) expression. Hum Mol Genet 26:2062-2075.

Freeman MR, Rowitch DH (2013) Evolving concepts of gliogenesis: a look way back and ahead to the next 25 years. Neuron 80:613-623.

Gründer S, Müller A, Ruppersberg JP (2001) Developmental and cellular expression pattern of epithelial sodium channel alpha, beta and gamma subunits in the inner ear of the rat. Eur J Neurosci 13:641-648.

Gulley RL, Reese TS (1976) Intercellular junctions in the reticular lamina of the organ of Corti. J Neurocytol 5:479-507.

Huang AY-S, Woo J, Sardar D, Lozzi B, Bosquez Huerta NA, Lin C-CJ, Felice D, Jain A, Paulucci-Holthauzen A, Deneen B (2020) Region-specific transcriptional control of astrocyte function oversees local circuit activities. Neuron 106:992-1008.e9.

John Lin C-C, Yu K, Hatcher A, Huang T-W, Lee HK, Carlson J, Weston MC, Chen F, Zhang Y, Zhu W, Mohila CA, Ahmed N, Patel AJ, Arenkiel BR, Noebels JL, Creighton CJ, Deneen B (2017) Identification of diverse astrocyte populations and their malignant analogs. Nat Neurosci 20:396405.

Khakh BS, Deneen B (2019) The emerging nature of astrocyte diversity. Annu Rev Neurosci 42:187-207.

Lanjakornsiripan D, Pior BJ, Kawaguchi D, Furutachi S, Tahara T, Katsuyama Y, Suzuki Y, Fukazawa Y, Gotoh Y (2018) Layer-specific morphological and molecular differences in neocortical astrocytes and their dependence on neuronal layers. Nat Commun 9:1623.

Lesca G, Moizard M-P, Bussy G, Boggio D, Hu H, Haas SA, Ropers H-H, Kalscheuer VM, Des Portes V, Labalme A, Sanlaville D, Edery P, Raynaud M, Lespinasse J (2013) Clinical and neurocognitive characterization of a family with a novel MED12 gene frameshift mutation. Am J Med Genet A 161A:3063-3071.

López-Bigas N, Olivé M, Rabionet R, Ben-David O, Martínez-Matos JA, Bravo O, Banchs I, Volpini V, Gasparini P, Avraham KB, Ferrer I, Arbonés ML, Estivill X (2001) Connexin 31 (GJB3) is expressed in the peripheral and auditory nerves and causes neuropathy and hearing impairment. Hum Mol Genet 10:947-952.

Loponen H, Ylikoski J, Albrecht JH, Pirvola U (2011) Restrictions in cell cycle progression of adult vestibular supporting cells in response to ectopic cyclin D1 expression. PLoS One 6:e27360.

Manalo JM, Liu H, Ding D, Hicks J, Sun H, Salvi R, Kellems RE, Pereira FA, Xia Y (2020) Adenosine A2B receptor: A pathogenic factor and a therapeutic target for sensorineural hearing loss. FASEB J 34:15771-15787.

Mills DM (2003) Differential responses to acoustic damage and furosemide in auditory brainstem and otoacoustic emission measures. J Acoust Soc Am 113:914-924.

Mittal R, Aranke M, Debs LH, Nguyen D, Patel AP, Grati M, Mittal J, Yan D, Chapagain P, Eshraghi AA, Liu XZ (2017) Indispensable role of ion channels and transporters in the auditory system. J Cell Physiol 232:743-758.

Muller U (2008) Cadherins and mechanotransduction by hair cells. Curr Opin Cell Biol 20:557-566. 
Nagai J, Yu X, Papouin T, Cheong E, Freeman MR, Monk KR, Hastings MH, Haydon PG, Rowitch D, Shaham S, Khakh BS (2021) Behaviorally consequential astrocytic regulation of neural circuits. Neuron 109:576-596.

Nin F, Yoshida T, Sawamura S, Ogata G, Ota T, Higuchi T, Murakami S, Doi K, Kurachi Y, Hibino H (2016) The unique electrical properties in an extracellular fluid of the mammalian cochlea; their functional roles, homeostatic processes, and pathological significance. Pflugers Arch 468:1637-1649.

Oesterle EC, Campbell S, Taylor RR, Forge A, Hume CR (2008) Sox2 and JAGGED1 expression in normal and drug-damaged adult mouse inner ear. J Assoc Res Otolaryngol 9:65-89.

Ohlemiller KK (2009) Mechanisms and genes in human strial presbycusis from animal models. Brain Res 1277:70-83.

Patuzzi R (2011) Ion flow in stria vascularis and the production and regulation of cochlear endolymph and the endolymphatic potential. Hear Res 277:4-19.

Petrov T, Howarth AG, Krukoff TL, Stevenson BR (1994) Distribution of the tight junction-associated protein ZO-1 in circumventricular organs of the CNS. Brain Res Mol Brain Res 21:235-246.

Rocha PP, Bleiss W, Schrewe H (2010a) Mosaic expression of Med12 in female mice leads to exencephaly, spina bifida, and craniorachischisis. Birth Defects Res A Clin Mol Teratol 88:626-632.

Rocha PP, Scholze M, Bleiss W, Schrewe H (2010b) Med12 is essential for early mouse development and for canonical Wnt and Wnt/PCP signaling. Development 137:2723-2731.

Rozengurt N, Lopez I, Chiu CS, Kofuji P, Lester HA, Neusch C (2003) Time course of inner ear degeneration and deafness in mice lacking the Kir4.1 potassium channel subunit. Hear Res 177:71-80.

Rubinato E, Rondeau S, Giuliano F, Kossorotoff M, Parodi M, Gherbi S, Steffan J, Jonard L, Marlin S (2020) MED12 missense mutation in a three-generation family. Clinical characterization of MED12-related disorders and literature review. Eur J Med Genet 63:103768.

Samaco RC, Fryer JD, Ren J, Fyffe S, Chao H, Sun Y, Greer JJ, Zoghbi HY, New JL (2008) A partial loss of function allele of methyl-CpG-binding protein predicts a human neurodevelopmental syndrome. Hum Mol Genet 17:1718-1727.
Schuknecht HF, Gacek MR (1993) Cochlear pathology in presbycusis. Ann Otol Rhinol Laryngol 102:1-16.

Schwartz CE, Tarpey PS, Lubs HA, Verloes A, May MM, Risheg H, Friez MJ, Futreal PA, Edkins S, Teague J, Briault S, Skinner C, Bauer-Carlin A, Simensen RJ, Joseph SM, Jones JR, Gecz J, Stratton MR, Raymond FL, Stevenson RE (2007) The original Lujan syndrome family has a novel missense mutation (p.N1007S) in the MED12 gene. J Med Genet 44:472477.

Stolt CC, Rehberg S, Ader M, Lommes P, Riethmacher D, Schachner M, Bartsch U, Wegner M (2002) Terminal differentiation of myelin-forming oligodendrocytes depends on the transcription factor Sox10. Genes Dev 16:165-170.

Trowe MO, Maier H, Petry M, Schweizer M, Schuster-Gossler K, Kispert A (2011) Impaired stria vascularis integrity upon loss of E-cadherin in basal cells. Dev Biol 359:95-107.

Ueberfuhr MA, Fehlberg H, Goodman SS, Withnell RH (2016) A DPOAE assessment of outer hair cell integrity in ears with age-related hearing loss. Hear Res 332:137-150.

Vogl MR, Reiprich S, Küspert M, Kosian T, Schrewe H, Nave K-A, Wegner M (2013) Sox 10 cooperates with the mediator subunit 12 during terminal differentiation of myelinating glia. J Neurosci 33:6679-6690.

Vulto-van Silfhout AT, de Vries BBA, van Bon BWM, Hoischen A, Ruiterkamp-Versteeg M, Gilissen C, Gao F, van Zwam M, Harteveld CL, van Essen AJ, Hamel BCJ, Kleefstra T, Willemsen MAAP, Yntema HG, van Bokhoven H, Brunner HG, Boyer TG, de Brouwer APM (2013) Mutations in MED12 cause X-linked Ohdo syndrome. Am J Hum Genet 92:401-406.

Wan G, Corfas G, Stone JS (2013) Inner ear supporting cells: rethinking the silent majority. Semin Cell Dev Biol 24:448-459.

Wangemann P (2006) Supporting sensory transduction: cochlear fluid homeostasis and the endocochlear potential. J Physiol 576:11-21.

Watanabe K, Takeda K, Katori Y, Ikeda K, Oshima T, Yasumoto K, Saito H, Takasaka T, Shibahara S (2000) Expression of the Sox10 gene during mouse inner ear development. Brain Res Mol Brain Res 84:141-145. 